\title{
A COMPACTUM THAT CANNOT BE AN ATTRACTOR OF A SELF-MAP ON A MANIFOLD
}

\author{
BERND GÜNTHER
}

(Communicated by James E. West)

\begin{abstract}
A one-dimensional compactum (in fact, a certain solinoid) is constructed, such that there does not exist a self-map on a manifold having this compactum as attractor.
\end{abstract}

\section{The COUNTEREXAMPLE}

In [2] it is shown that a finite-dimensional compactum can be an attractor of a flow on a manifold if and only if it has the shape of a finite polyhedron. Here a flow is a continuous mapping $X \times \mathbb{R} \rightarrow X$ satisfying the usual functional equation [5, Chapter $4, \S 7$, Theorem 12], and $X$ is a topological manifold. If $\mathbb{R}$ is replaced by the integers $\mathbb{Z}$ we simply get a cyclic group of homeomorphisms, generated by some element $f: X \approx X$. We may generalize still further and consider arbitrary self-maps $f: X \rightarrow X$ instead of homeomorphisms. A compact subset $A \subseteq X$ with $f(A)=A$ is an attractor of $f$ if there is a neighborhood $U$ of $A$ in $X$, such that $f(U) \subseteq U$ and for each neighborhood $V$ of $A$ in $X$ there is $n \in \mathbb{N}$ with $f^{n}(U) \subseteq V$; this implies $f^{m}(U) \subseteq V$ for all $m \geq n$. In this situation the classification of attractors seems to be much more complicated than in [2], but at least we are able to produce an example of a one-dimensional compactum that can never be an attractor of a self-map.

It is well known that the dyadic solenoid is an attractor of a homeomorphism of a three-dimensional manifold (see [4; 2, Example 3]). The dyadic solenoid is the limit of an inverse sequence of circles $S_{(n)}^{1}=\{z \in \mathbb{C}|| z \mid=1\}$, and the bonding map $g_{n}: S_{(n+1)}^{1} \rightarrow S_{(n)}^{1}$ is given by $g_{n}(z)=z^{2}$. Of course, we might as well choose different bonding maps such as $g_{n}(z)=z^{\nu_{n}}$, where $\left(\nu_{n}\right)_{n \in \mathbb{N}}$ is an arbitrary sequence of integers. The compacta obtained in this way are generalized solenoids.

Theorem 1. The generalized solenoid obtained from a sequence of pairwise relatively prime integers $\nu_{n}$ cannot be an attractor of a self-map on a manifold.

Received by the editors July 7, 1992.

1991 Mathematics Subject Classification. Primary 55P55, 57N25, 54H20, 58F12.

Key words and phrases. Dynamical systems, attractors, shape, solenoid. 
For the proof the following lemma is needed.

Lemma 1. We suppose $A$ is an attractor of $f: X \rightarrow X$ and denote by $\varphi:=$ $\check{H}^{*}\left(f_{\mid A}\right): \check{H}^{*}(A) \rightarrow \check{H}^{*}(A)$ the induced homomorphism of Čech cohomology groups with coefficients in $\mathbb{Z}$. Then $\check{H}^{*}(A)$ contains a finitely generated, $\varphi$ invariant subgroup $G$ with $\bigcup_{n=1}^{\infty} \varphi^{-n}(G)=\check{H}^{*}(A)$.

Proof. Let $U$ be a domain of attraction as above. $A$ is the inverse limit of a sequence of finite polyhedra $P_{n}$ with projections $g_{n}: A \rightarrow P_{n}$ [3, Chapter I, $\S 5.2$, Corollary 4]. The inclusion map $i: A \hookrightarrow U$ can be factored over one of these $g_{n}$ up to homotopy [3, Chapter I, $\S 5.3$, Theorem 9], because the interior of $U$ is a manifold and hence an ANR. We may assume $n=1: i \simeq h g_{1}$ for a suitable map $h: P_{1} \rightarrow U$ and set

$$
G^{\prime}:=\operatorname{im} \check{H}^{*}\left(g_{1}\right) \quad \text { and } \quad G:=\bigcap_{m=0}^{\infty} \varphi^{-m}\left(G^{\prime}\right) .
$$

Any given element $\xi \in \check{H}^{*}(A)$ can be written $\xi=\check{H}^{*}\left(g_{k}\right)(\eta)$ for some $k$ [3, p. 128]. There is a neighborhood $V$ of $A$ in $X$ and a map $g_{k}^{\prime}: V \rightarrow P_{k}$ extending $g_{k}$. Let $n$ be such that $f^{m}(U) \subseteq V$ for all $m \geq n$. Then $g_{k}^{\prime} f^{m} h g_{1}$ : $X \rightarrow P_{k}$ is homotopic to $g_{k} f^{m}$ and consequently

$$
\varphi^{m}(\xi)=\check{H}^{*}\left(g_{k} f^{m}\right)(\eta)=\check{H}^{*}\left(g_{1}\right) \check{H}^{*}\left(g_{k}^{\prime} f^{m} h\right)(\eta) \in G^{\prime} .
$$

This means $\varphi^{m} \varphi^{n}(\xi) \in G^{\prime}$ for all $m \geq 0$ and therefore $\varphi^{n}(\xi) \in G$.

Proof of Theorem 1. We suppose the contrary and denote by $H$ the first Čech cohomology group of our solenoid; it is isomorphic to the subgroup of $\mathbb{Q}$ consisting of all numbers $m / \nu_{1} \cdots \nu_{n}$ with $m \in \mathbb{Z}, n \in \mathbb{N}$. Let $G$ be the subgroup of $H$ from Lemma 1; it must be cyclic with a generator $M / \nu_{1} \cdots \nu_{N}$. The homomorphism $\varphi: H \rightarrow H$ must be of the form $\varphi(r)=r p / q$. We choose a number $k>N$ such that $\nu_{k}$ is relatively prime to $p$. Then for all $m \geq 0$ we have $\varphi^{m}\left(1 / \nu_{k}\right) \notin G$, contradicting Lemma 1 .

Remark. The subgroup $G$ in Lemma 1 behaves like an "attractor" of the endomorphism $\varphi$ : each element of $\check{H}^{*}(A)$ is eventually moved into $G$. This puts restrictions on the map $f_{\mid A}: A \rightarrow A$; in the case of the dyadic solenoid $\varphi$ must be multiplication by a number $2^{m}$ with $m>0$. Observing $\check{H}^{1}(A)=$ HTop $\left(A, S^{1}\right)$ one can deduce that in the shape category $f_{\mid A}$ coincides with $\vartheta^{m}$, where $\vartheta$ is the shift map of the dyadic solenoid [4, Lemma 29].

Note. For expanding attractors of diffeomorphisms Theorem 1 can also be obtained by using Williams's characterization [6] and the classification of solenoids [1, p. 154].

\section{REFERENCES}

1. K. Borsuk, Theory of shape, Monografie Matematyczne, vol. 59, Polish Scientific Publishers, 1975.

2. B. Günther and J. Segal, Every attractor of a flow on a manifold has the shape of a finite polyhedron, Proc. Amer. Math. Soc. 119 (1993), 321-329.

3. S. Mardešić and J. Segal, Shape theory, North-Holland Math. Library, vol. 26, North-Holland, Amsterdam, 1982. 
4. R. M. Schori, Chaos: An introduction to some topological aspects, Continuum Theory and Dynamical Systems (Morton Brown, ed.), Contemp. Math., vol. 117, Amer. Math. Soc., Providence, RI, 1991, pp. 149-161.

5. E. H. Spanier, Algebraic topology, McGraw-Hill, New York, 1966.

6. R. F. Williams, Classification of one-dimensional attractors, Proc. Sympos. Pure Math., vol. 14, Amer. Math. Soc., Providence, RI, 1970, pp. 341-361.

Helpertseestrasse 21, 6052 Mühlheim, Germany

E-mail address: 100034 .3630@CompuServe.COM 